\title{
Evaluation of atrial electromechanical conduction delay in case of hemodynamically insignificant rheumatic heart disease: A tissue Doppler study
}

\author{
Metin Cagdas, Yalcin Velibey, Tolga Sinan Guvenc, Baris Gungor, Ozge Guzelburc, \\ Nazmi Calik, Murat Ugur, Ahmet Ilker Tekkesin, Kadir Gurkan, Mehmet Eren \\ Department of Cardiology, Training and Research Hospital, Siyami Ersek Thoracic \\ and Cardiovascular Surgery Center, Istanbul, Turkey
}

\begin{abstract}
Background: Atrial electromechanical delay (AEMD) that reflects delayed conduction may show us the clinical reflection of pathological changes in the atria. The main objective of the present study is to investigate AEMD in patients who had previous rheumatic carditis but without hemodynamically significant valvular disease.

Methods: A total of 40 patients, previously diagnosed as rheumatic carditis but without significant valvular stenosis/regurgitation and atrial enlargement; and 39 age-and-sex matched controls were enrolled for the present study. Parameters of AEMD (lateral mitral annulus electromechanical delay, septal mitral annulus electromechanical delay and lateral tricuspid annulus electromechanical delay) were measured with tissue Doppler echocardiography and left intra-atrial and inter-atrial conduction times were calculated accordingly. A 24 h ambulatory Holter monitoring was used in both groups to detect atrial fibrillation episodes and quantify atrial extrasystoles.
\end{abstract}

Results: Parameters of AEMD, including left intra-atrial and inter-atrial conduction times of subjects in the study group were longer compared to the control group $(23.7 \pm 7.0 \mathrm{vs.} 18.3 \pm 6.2)$. Conclusions: Increased AEMD is observed in patients with previous rheumatic carditis and no significant valvular stenosis/regurgitation and atrial enlargement, which may partly explain the increased incidence of atrial fibrillation observed in these patients. (Cardiol J 2015; 22, 6: 683-690)

Key words: atrial electromechanical conduction delay, hemodynamically insignificant rheumatic heart disease, echocardiography

\section{Introduction}

Rheumatic valvular disease (RVD) still has an important place among heart diseases in less developed and developing societies. Approximately 15 million people are estimated to be suffering from rheumatic valvular disease worldwide [1,2]. In regions with a high prevalence of acute rheumatic fever (ARF), echocardiographic screening for the detection of RVD is still recommended by the World Health Organization [3].

Address for correspondence: Yalcin Velibey, MD, Dr. Siyami Ersek Hospital, Tibbiye Str. No: 25, Uskudar, Istanbul, Turkey, tel: +902164445257, fax: +902163379719, e-mail: dr_yalchin_dr@yahoo.com.tr

Received: 28.05.2015 Accepted: 22.06.2015 
The incidence of atrial fibrillation (AF) is increased in patients with RVD and it can be as high as $70 \%$ when accompanying mitral regurgitation, mitral stenosis or tricuspid regurgitation are present [4]. The basic mechanism of valvularoriginated $\mathrm{AF}$ is atrial remodeling, which involves histo-pathological and electrophysiological changes induced by the increase in atrial volume and pressure. Still, an increased incidence of AF is observed in patients with previous rheumatic carditis (RC) without significant valve stenosis/ /regurgitation and pressure/volume overload. The exact cause for this increase is not known, but it was previously suggested that chronic atrial inflammation accompanying previous rheumatic fever (RF) could create a substrate for $\mathrm{AF}[5,6]$.

Delayed inter- and intra-atrial conduction time (ACT) reflects heterogeneity of atrial conductivity, which is a known risk factor for AF. The predictive value of ACTs for the development of AF, whether measured invasively with electrophysiologic study or noninvasively with echocardiography, has been established in previous studies $[7,8]$. The prolongation of ACT is linked to a variety of mechanisms, including atrial dilatation and remodeling, chronic inflammation within the atrial myocardium, and atrial fibrosis. Therefore, ACT prolongation could act as an intermediary mechanism linking RF-related changes in atrium (including chronic inflammation and fibrosis) with the development of AF.

In the present study, we aimed to investigate the duration of ACT in RVD patients without significant valvular stenosis/regurgitation or atrial enlargement and to compare these findings with healthy controls. In addition, we investigated the value of ACT duration as a predictor for development of AF in patients with previous RF.

\section{Methods}

\section{Study design and patient selection}

Patients who admitted to institutional outpatient clinic between January 2010 and January 2012 with a previous diagnosis of RF were enrolled in the present study. The inclusion criteria included 1) At least one previous documented RF episode in which the diagnosis was established according to Jones or Modified Jones criteria and documented signs for cardiac involvement during this episode (new valvular murmur, signs of heart failure, etc.) or 2) Rheumatic valve involvement at follow-up echocardiograms in patients with a previous documented RF episode. All these patients were using or had previously used depot penicillin monthly for secondary prophylaxis for a long time. All systemic diseases or disorders and structural cardiac pathologies that could have affected electromechanical conduction delay times were evaluated meticulously in order to obtain more objective results. Patients under 18 years of age, those with a history of previous myocarditis and/or pericarditis not attributable to ARF episode, right and/or left atrial (LA) enlargement during screening echocardiography, moderate or severe mitral, tricuspid, or aortic valve insufficiency, mild, moderate, or severe stenosis in any one of mitral, aortic, tricuspid and pulmonary valves, those with congenital cardiac defects (including those with a previous operation for congenital heart disease), coronary artery disease, previous episode of unstable angina/myocardial infarction, previous coronary revascularization, any type of cardiomyopathy, systemic/pulmonary hypertension, diabetes mellitus, any degree of diastolic dysfunction during screening echocardiography, inadequate image quality during screening echocardiography, severe systemic disease (chronic kidney disease, chronic liver disease, autoimmune disease, anemia, hyperthyroidism or hypothyroidism, malignancy and electrolyte disorders) that could have led to diastolic dysfunction or that could have affected electromechanical conduction delay times were excluded from the study. In addition, patients who underwent ablation therapy due to tachyarrhythmia, were taking antiarrhythmic medications, had a resting pulse rate higher than $90 \mathrm{bpm}$ or lower than $60 \mathrm{bpm}$ were excluded from the study. A screening echocardiogram was commenced prior to enrolment to check the presence of exclusion criteria. A total of 76 subjects with previous RC history were evaluated. Thirty-six subjects were excluded because of having one or more exclusion criteria that we mentioned above and finally, 40 patients who fulfilled all criteria were enrolled. Thirty-nine asymptomatic healthy volunteers without medical history (of any systemic disease), in whom no cardiovascular pathology was detected as a result of treadmill exercise testing, electrocardiographic (ECG), echocardiographic, laboratory and clinical assessment, were included in the control group. None of these people had a history of RC and they had normal valvular structure echocardiographically. Non-invasive diagnostic methods were performed to obtain and exclude subclinical or hidden disorders in these asymptomatic patients. Because minimal mitral and/or aortic regurgitation present in the both groups does not lead to a structural and functional alteration in the LA, these minimal 
valvular pathologies will not, in theory, have an independent effect on atrial electromechanical delay (EMD) times. All participants included in the study were examined in the echocardiography laboratory of Dr Siyami Ersek Hospital between January 2010 and January 2012.

Clinical and demographic information was collected from all subjects at the time of enrolment. A baseline ECG and laboratory tests, including complete blood count and blood chemistry, were obtained, with the blood withdrawn after overnight fasting for the latter. The $\mathrm{p}$ wave duration (PWD) was measured from lead D II and an average of 3 measurements from consecutive beats was accepted as PWD. A PWD of more than $120 \mathrm{~ms}$ in the absence of LA enlargement (LA volume index $[\mathrm{LAVI}]<20 \mathrm{~mL} / \mathrm{m}^{2}$ ) was accepted as an interatrial block (IAB). To investigate the presence of atrial arrhythmias, including AF, a Holter device was used to digitally store 3-channel ECG for 24-h. These ECG recordings were analyzed offline using a computer terminal and compatible software to count the total number of atrial extrasystoles and other specific arrhythmias. All procedures were performed using standardized techniques.

This study was carried out according to the Principles of the Declaration of Helsinki, and written informed consent was obtained from all the patients. The study was approved by the institutional Ethics Committee.

\section{Echocardiographic measurements}

A standard transthoracic echocardiographic examination was performed on each patient by a single experienced cardiologist. Transthoracic echocardiographic images were obtained from the parasternal and apical 4, 3 and 2 chamber views with $2.5-3.5 \mathrm{MHz}$ phased-array transducer by using GE Vivid 7 machine (GE Vivid 7, GE Healthcare, Piscataway, NJ, USA). The examination was performed while the patient was lying down in the left lateral and supine positions; each patient was evaluated by M-mode, B-mode, color Doppler and tissue Doppler echocardiography. Single-lead ECG recordings were taken continuously throughout the examination. Echocardiography and ECG signals were recorded in harmony in order to obtain more objective measurements and not to have measurement errors. All measurements were made with the same probe in the same echocardiography device by a single experienced cardiologist. Left ventricular end-diastolic, end-systolic diameters and left atrium end-systolic diameters were measured with M-mode echocardiography from parasternal long axis view. Left atrial area which was measured in order to calculate the left atrial volume was recorded as the average of the widest area measured at 3 consecutive beats. Left atrial volume was calculated by using biplane Simpson method as recommended by the American Society of Echocardiography. LAVI was calculated in terms of $\mathrm{mL} / \mathrm{m}^{2}$ by dividing left atrial volume to body surface area. Mitral inflow velocity was assessed by pulsed-wave Doppler placed at the tip of the mitral valve from the apical 4-chamber window. Diastolic early (E) and late (A) peak flows were measured. Tissue Doppler echocardiography was performed by adjusting spectral pulsed Doppler signal filters until the Nyquist limit was $15-20 \mathrm{~cm} / \mathrm{s}$, with $3.5-4.0 \mathrm{MHz}$ frequency transducer, by using the minimal optimal gain. Sweep speed of the monitor was set $100 \mathrm{~mm} / \mathrm{s}$ so that the spectral images of myocardial velocities were optimal. Tissue Doppler spectral views were obtained by placing pulsed wave (PW) tissue Doppler cursor in the apical 4-chamber view window to the left ventricular mitral lateral and septal annulus and right ventricular tricuspid lateral annulus, respectively. PW cursor was aligned as parallel as possible to the myocardial segment of interest to ensure the optimal angle of imaging. Myocardial peak systolic (S), early (E') and late (A') diastolic peak velocities were obtained from these regions. Atrial EMD delay was defined as the time (PA') elapsed from the beginning of the $P$ wave in lead D II in the ECG to the beginning of the A' wave in tissue Doppler trace for lateral mitral annulus (PA' lateral EMD), septal mitral annulus (PA' septal EMD) and lateral tricuspid annulus (PA' tricuspid EMD) in terms of millisecond. A flow diagram for the study is provided in Figure 1.

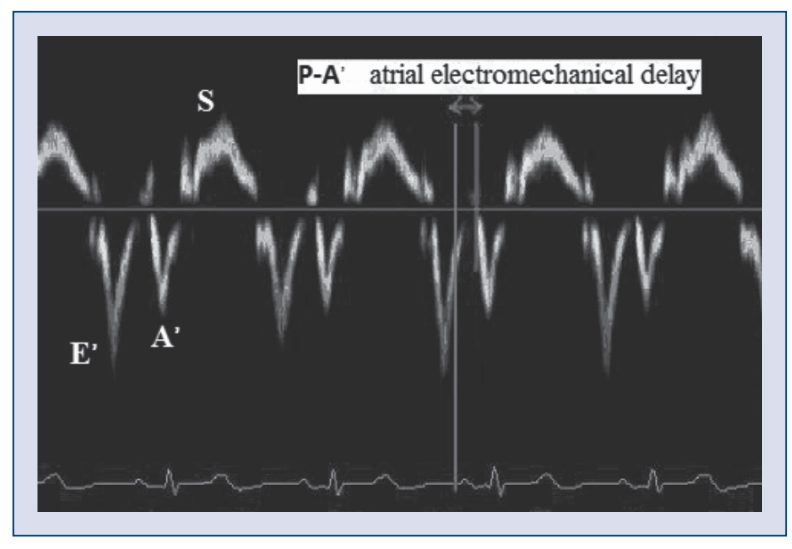

Figure 1. Measurement of atrial electromechanical conduction delay via tissue Doppler echocardiography. 
Table 1. Baseline demographics, hemodynamic parameters, hemoglobin, thyroid stimulating hormone levels and atrial extrasystole (AES) incidence in 24-h rhythm Holter recordings of the 77 study subjects (patient and control group), categorized with respect to the center of origin.

\begin{tabular}{lccc}
\hline Variables & $\begin{array}{c}\text { Patient group } \\
\text { (mean } \pm \text { SD) }\end{array}$ & $\begin{array}{c}\text { Control group } \\
\text { (mean } \pm \text { SD) }\end{array}$ & P \\
\hline Patients & 40 & 39 & - \\
Age [year] & $34.8 \pm 9.1$ & $31.3 \pm 10.1$ & 0.13 \\
Male & $18.4 \%$ & $20.2 \%$ & 0.15 \\
Body surface area [m $]$ & $1.69 \pm 0.15$ & $1.69 \pm 0.21$ & 0.88 \\
Heart rate [bpm] & $74.3 \pm 9.4$ & $76 \pm 6.7$ & 0.3 \\
Systolic blood pressure [mm Hg] & $103.5 \pm 9.4$ & $105.6 \pm 8.1$ & 0.29 \\
Diastolic blood pressure [mm Hg] & $66.2 \pm 9$ & $65.9 \pm 8.1$ & 0.88 \\
Hemoglobin [mg/dL] & $13.2 \pm 1.3$ & $13.7 \pm 1.2$ & 0.13 \\
TSH [IU/mL] & $2.3 \pm 1.0$ & $2.4 \pm 0.96$ & 0.13 \\
AES in 24-h rhythm Holter ECG & $357.63 \pm 2,088.30$ & $0.79 \pm 1.13$ & $<0.01$ \\
P wave duration [ms] & $90.47 \pm 10.31$ & $71.48 \pm 12.44$ & $<0.001$ \\
Interatrial block [\%] & 0 & 0 & 1 \\
AES in 24-h rhythm Holter ECG (after exclusion of outliers) & $18.89 \pm 26.71$ & $0.79 \pm 1.13$ & $<0.01$ \\
\hline
\end{tabular}

SD — standard deviation; TSH — thyroid stimulating hormone; ECG — electrocardiography

Values were averaged over 3 consecutive beats. To obtain a perfect synchronization all measurements of every patient were remade and the same results were obtained. The difference between PA' lateral EMD and PA' tricuspid EMD was defined as inter-atrial conduction time, and the difference between PA' lateral EMD and PA' septum EMD was defined as left intra-atrial conduction time. For all measurements, recommendations from the American Society of Echocardiography were used where possible.

\section{Statistical analysis}

SPSS 15.0 software was used for statistical analysis of the data obtained from the study. Continuous variables were presented as mean \pm standard deviation while categorical data were given as percentage of total. One-way KolmogorovSmirnov test was used to determine the pattern of distribution for continuous variables. For continuous variables with a normal distribution, Student's t-test was used to compare groups, while MannWhitney U test was used when the distribution was not normal. For categorical variables, $\chi^{2}$ and Fisher's exact tests were used as appropriate. Correlations between continuous variables were analyzed with Pearson or Spearman correlation tests, with the latter used when the distribution of one of the parameters within the group was not normal. For all comparisons, a p value lower than 0.05 was accepted as significant.

\section{Results}

Demographic, and clinical data regarding the study and control groups are given in Table 1 . There were no statistically significant differences between groups regarding the demographic or clinical variables. PWD was significantly higher in the study group $(90.47 \pm 10.31$ vs. $71.48 \pm 12.44$, $\mathrm{p}<0.001$ ), but no subjects in both groups had a PWD > $120 \mathrm{~ms}$ and therefore had no IAB. Echocardiographic variables, such as left ventricular dimensions, wall thickness, ejection fraction, left atrial volume, LAVI, E, A, E', A' and E/E' were also similar between two groups. There was a positive correlation between LAVI and age of subjects $(\mathrm{r}=0.336, \mathrm{p}<0.01)$. Aortic valve involvement was present in $42 \%(n=16)$ of cases in the study group. No correlation was found between LAVI and the number of affected valves $(r=0.11$, $\mathrm{p}=0.32)$ (Table 2).

Indices of atrial EMD, including PA' lateral EMD, PA' septal EMD and PA' tricuspid EMD; left intra-and inter-atrial conduction times, measured by echocardiography, of the patients enrolled in the study are presented in Table 3. Atrial EMD, left intra- and inter-atrial conduction times were 
Table 2. Two dimensional echocardiographic parameters of study subjects.

\begin{tabular}{lccc}
\hline Variables & Patient group (mean \pm SD) & Control group (mean \pm SD) & $P$ \\
\hline LVEDD [mm] & $44.5 \pm 1.6$ & $44.9 \pm 2.2$ & 0.37 \\
LVESD [mm] & $25.4 \pm 2.0$ & $25.9 \pm 2.2$ & 0.31 \\
IVS [mm] & $8.07 \pm 0.88$ & $8.1 \pm 0.99$ & 0.91 \\
PW [mm] & $7.8 \pm 0.75$ & $7.7 \pm 0.78$ & 0.57 \\
EF [\%] & $65.6 \pm 3.1$ & $65.1 \pm 2.5$ & 0.47 \\
LAVol [mL] & $37.4 \pm 8.4$ & $35.2 \pm 6.5$ & 0.2 \\
LAVI [mL/m²] & $22.3 \pm 3.6$ & $21.5 \pm 3.4$ & 0.3 \\
E [m/s] & $0.95 \pm 0.19$ & $0.95 \pm 0.18$ & 0.98 \\
A [m/s] & $0.63 \pm 0.14$ & $0.61 \pm 0.15$ & 0.62 \\
$E^{\prime}[\mathrm{cm} / \mathrm{s}]$ & $14.5 \pm 2.5$ & $14.8 \pm 2.3$ & 0.66 \\
A $^{\prime}[\mathrm{cm} / \mathrm{s}]$ & $8.8 \pm 2.5$ & $8.9 \pm 2.3$ & 0.88 \\
E/E' & $6.6 \pm 1.5$ & $6.5 \pm 1.3$ & 0.63 \\
\hline
\end{tabular}

SD - standard deviation; LVEDD - left ventricular end-diastolic dimension; LVESD - left ventricular end-systolic dimension; IVS - interventricular septum; PW - posterior wall; EF — ejection fraction; LAVol — left atrial volume; LAVI — left atrial volume index

Table 3. Atrial electromechanical delay (EMD), left intra-atrial and inter-atrial conduction times of the study subjects.

\begin{tabular}{lccc}
\hline Variables & Patient group (mean \pm SD) & Control group (mean \pm SD) & P \\
\hline PA' lateral EMD [ms] & $89.4 \pm 10.8$ & $68.6 \pm 14.9$ & $<0.001$ \\
PA' septal EMD [ms] & $65.7 \pm 9.1$ & $50.2 \pm 11.4$ & $<0.001$ \\
PA' tricuspid EMD [ms] & $50.74 \pm 7.0$ & $41.5 \pm 9.0$ & $<0.001$ \\
Intra-atrial conduction & $23.7 \pm 7.0$ & $18.3 \pm 6.2$ & $<0.001$ \\
time [ms] & $38.7 \pm 12.1$ & $27.4 \pm 8.2$ & $<0.001$ \\
Inter-atrial conduction & & & \\
time [ms] & & & \\
\hline
\end{tabular}

SD — standard deviation; $\mathrm{PA}^{\prime}$ lateral EMD — lateral mitral annulus EMD; $\mathrm{PA}^{\prime}$ septal EMD — septal mitral annulus EMD; $\mathrm{PA}$ ' tricuspid EMD — lateral tricuspid annulus EMD

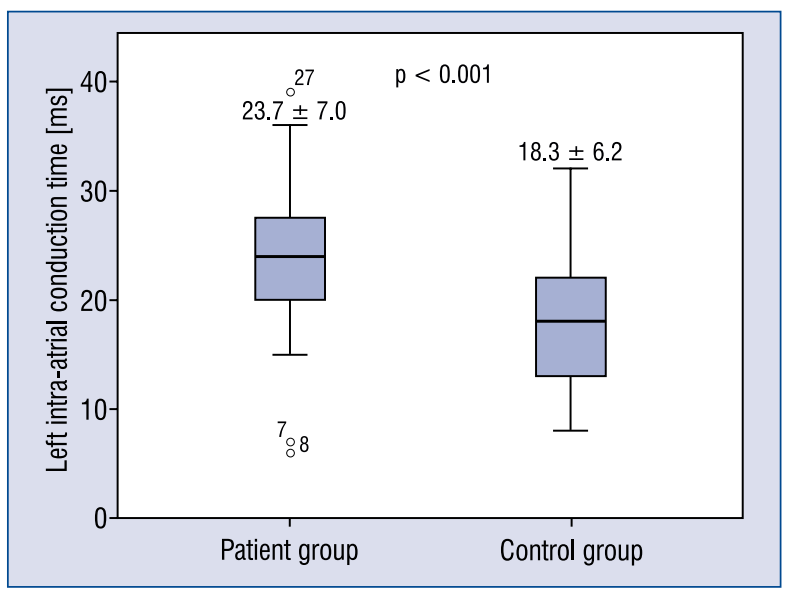

Figure 2. Box-plot representations for left intra-atrial conduction time in study and control groups. The difference between groups is statistically significant at 0.001 level.

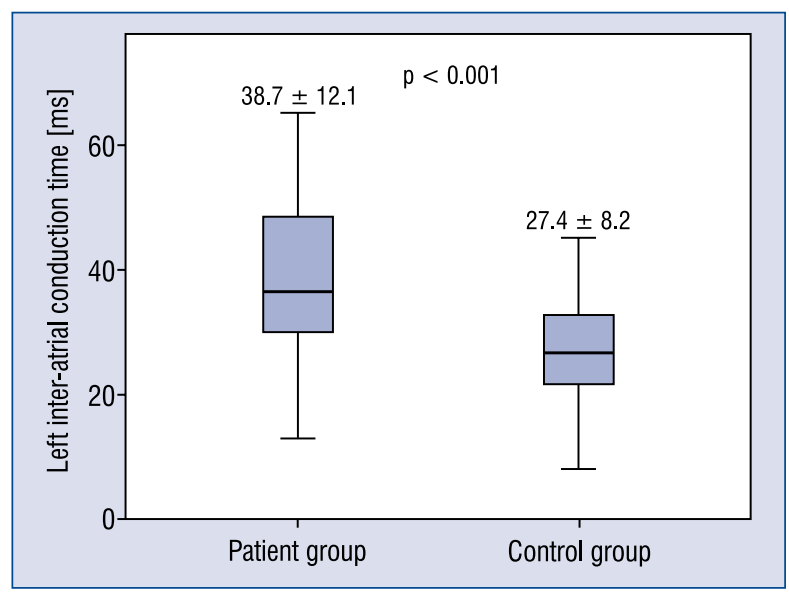

Figure 3. Box-plot representations for left inter-atrial conduction time in study and control groups. The difference between groups is statistically significant at 0.001 level. 
prolonged in the study group compared to the healthy volunteers $(\mathrm{p}<0.001)$ (Table 3 , Figs. 2, 3). There was no significant correlation between LAVI and PA' lateral EMD $(\mathrm{r}=0.057, \mathrm{p}=0.614), \mathrm{PA}^{\prime}$ septal EMD $(r=0.040, p=0.724)$, PA' tricuspid $\operatorname{EMD}(r=0.099, p=0.388)$, left intra-atrial conduction time $(\mathrm{r}=0.062, \mathrm{p}=0.587)$ and inter-atrial conduction time $(\mathrm{r}=0.11, \mathrm{p}=0.924)$. PWD had a significant, moderate-to-well correlation with both left intra-atrial $(r=0.51, \mathrm{p}<0.001$ for the study group and $\mathrm{r}=0.71, \mathrm{p}<0.001$ for the control group) and inter-atrial $(\mathrm{r}=0.72, \mathrm{p}<0.001$ for the study group and $r=0.73, p<0.001$ for the control groups) conduction times. The total count of atrial extrasystoles on $24 \mathrm{~h}$ Holter monitoring was significantly higher in the study group compared to the control group (357.63 \pm $\pm 2,088.30$ vs. $0.79 \pm 1.13, \mathrm{p}<0.001$ ), and the statistical significance remained even when one patient with a high atrial extrasystole count $(12,891)$ was excluded from the analysis $(18.89 \pm 26.71 \mathrm{vs}$. $0.79 \pm 1.13, \mathrm{p}<0.001)$. There was a significant correlation between intra-atrial conduction time and atrial extrasystoles (AES) count in the study group $(\mathrm{p}=0.025, \mathrm{r}=0.42)$, but the statistical significance was lost when one outlier patient was excluded from analysis $(\mathrm{p}=0.21, \mathrm{r}=0.25)$. One episode of $\mathrm{AF}$ was observed in 1 patient in the study group.

\section{Discussion}

Humoral and cellular inflammation within the endocardium, myocardium, pericardium and valvular structures which is caused by an autoimmune response, forms the basis of ARC. Following the acute episode, a chronic inflammatory reaction which is characterized by infiltration of target tissues by $T$ cells and macrophages persist for years and eventually causes deformation of valvular structures, vacuolar degeneration within the atrial myocytes, interstitial fibrosis, and an increase in adipocytes [6]. Both the formation and the propagation of electrical impulses are negatively affected by increased interstitial fibrosis and cellular degeneration. These changes within the atrium cause a decrease in the number of L-type calcium channels and reduced calcium influx that affects impulse generation and transmission. These changes are important electrophysiological precursors for the development of $\mathrm{AF}$, as shown with many pre-clinical and clinical studies [9-12]. In patients with pure rheumatoid mitral disease, subjects with AF had higher left atrial volumes than those in sinus rhythm, and the degree of underlying valvular pathology did not correlate with LA volume. In addition, all patients with AF had histopathological changes in atrial myocardium, such as vacuolar degeneration and interstitial fibrosis, while the same changes were observed in $56 \%$ of patients with normal sinus rhythm [5]. These findings suggest that chronic inflammation per se plays a role for the development of $\mathrm{AF}$, which is independent of underlying valvular pathology [6].

Indicators of electromechanical heterogeneity along the atria reflect the pathological changes within the atrium and also indicate an increased risk for AF development [1, 12]. Although the gold standard measure for atrial EMD is direct measurement with an electrophysiological study (EPS), the invasive nature of this modality makes it impractical to be used to determine ACTs. Instead, tissue Doppler echocardiography allows noninvasive measurement of ACTs with an acceptable accuracy when compared to EPS [8]. Prolongation of ACTs is observed in patients with various conditions that pose an increased tendency to AF, such as paroxysmal AF, mitral stenosis or systemic sclerosis [13-15]. Left atrial dilatation is undoubtedly a major cause for this increase in ACTs. However, $\mathrm{ACT}$ prolongation can be observed even in the absence of LA dilatation, suggesting presence of additional factors. Patients with systemic inflammatory diseases, such as systemic sclerosis or psoriasis vulgaris, have longer ACT duration and a tendency for AF development, suggesting a relationship between chronic inflammation and ACT $[14,15]$. Present study also shows that ACT prolongation is not solely related to LA dilatation, and is probably related to atrial inflammation. Unfortunately, cohort duration of follow up is inadequate in present study to show prolonged $\mathrm{ACT}$ as a risk factor for $\mathrm{AF}$ in patients with hemodynamically insignificant rheumatic carditis. However, in order to be able to say that prolongation of ACTs we obtained in patients with minimal rheumatic carditis may predict the development of AF, certainly follow-up period for years is required. Therefore, these patients were put under long-term follow-up for AF development.

Prolongation of PWD in the study group, as discussed later, further reinforces these findings. We hypothesize that chronic inflammation related to previous rheumatic involvement remains as the most plausible explanation for both findings, similar to systemic inflammatory diseases discussed before. Unfortunately, the duration of follow-up is inadequate in the present study to show that prolonged interatrial conduction time or PWD is a risk factor for $\mathrm{AF}$ in patients with previous $\mathrm{RC}$ but without significant valvulopathy and/or LA enlargement. 
Prolongation of PWD on surface ECG reflects abnormal interatrial conduction. A PWD duration of $120 \mathrm{~ms}$ of more in the presence of normal $P$ wave axis is termed a first degree IAB, while the presence of a negative terminal deflection on inferior leads in the presence of a PWD $>120 \mathrm{~ms}$ is termed a third degree IAB [16]. Both conditions are associated with an increased frequency of atrial tachyarrhythmias, including AF [17]. Although the frequency of IAB is closely related to the presence of LA enlargement, IAB can be seen in subjects with normal LA dimensions, and presence of IABs are related with abnormal LA function $[16,18]$. In the present study, we found prolonged PWD in study group, albeit none of the subjects had IAB. This finding, taken together with the prolongation of interatrial conduction time and the good correlation between PWD and interatrial conduction time - further supports the presence of conduction abnormalities in LA after RC, even in the absence of LA pressure or volume overload.

Atrial extrasystoles serve as a trigger for various supraventricular tachycardias, including $\mathrm{AF}$ [19]. Patients with increased number of AES on 24-h monitoring have an increased risk for developing $\mathrm{AF}$ [20]. We observed in this present study that patients with hemodynamically insignificant RC have more AES compared to healthy volunteers $(\mathrm{p}<0.01)$, and there is a tendency for more ACTs in those with more pronounced prolongation of intraatrial conduction time $(\mathrm{p}=0.025, \mathrm{r}=0.42)$, but the significance is lost when one outlier is removed. We consider that this potential relationship between intra-atrial conduction time and AES frequency is particularly interesting considering the relationship between AES count and AF development. However, a definite link could not be shown in this study and this topic deserves further research to ascertain whether ACT prolongation is also a risk factor for AES.

Although there is not any structural cardiac pathology in patients with lone AF, atrial volume is higher than the normal population and pathologic mitral regurgitation, albeit minimal, is more frequent [21]. In addition, there are cause unclear atrial signs of active and chronic inflammation in lone AF [22]. When we take into account that rheumatic valve disease may be asymptomatic and there is not any specific echocardiographic findings to RVD, likelihood of minimal RC, which triggers the inflammation in the atria in some patients with lone $\mathrm{AF}$, is quite thought-provoking.

\section{Limitations of this study}

The main limitation of the present study is that this is a single center study and the size of the study population was small. Limited power of the present study does not allow evaluation of the relationship between $\mathrm{ACT}$ prolongation and $\mathrm{AF}$ in this patient cohort, although previous studies have established prolonged ACTs as a risk factor of AF. Therefore, further studies are needed to ascertain whether this prolongation translates into clinically meaningful results. Inflammation remains the most plausible explanation for ACT prolongation in $\mathrm{RC}$ patients, but this study was not designed to investigate the cause of ACT prolongation and further research is needed to ascertain this point. Although RC patients have prolonged ACTs and more frequent AES on $24 \mathrm{~h}$ Holter, prolonged intraatrial conduction time may not be the cause for this increase considering the possibility of collinearity. In addition, speckle tracking was not used to evaluate atrial electromechanical conduction delay and we could not perform EPS to confirm the results because it was not ethically possible.

\section{Conclusions}

Patients with RC but without significant valve involvement have increased ACTs despite normal LA volumes. This increase in ACTs can explain the increased frequency of $\mathrm{AF}$ in patients with $\mathrm{RC}$ even when significant valve disease and LA pressure/ /volume overload is not present. More research is needed to ascertain whether this prolongation of ACTs could serve as a clinical tool to determine the risk of $\mathrm{AF}$ in this patient group.

\section{Conflict of interest: None declared}

\section{References}

1. Carapetis JR, Steer AC, Mulholland EK, Weber M. The global burden of group A streptococcal diseases. Lancet Infect Dis, 2005; 5: 685-694.

2. Carapetis J, Parr J, Cherian T. Standardization of epidemiologic protocols for surveillance of post-streptococcal sequelae: Acute rheumatic fever, rheumatic heart disease and acute poststreptococcal glomerulonephritis. Department of Health and Human Services, National Institutes of Health, January 2006; p. 1-32.

3. Remeny B, Wilson N, Steer A et al. World heart federation criteria for echocardiographic diagnosis of rheumatic heart disease, an evidence-based guideline. Nature Rev Cardiol, 2012; 9: 297-309.

4. Diker E, Aydogdu S, Ozdemir M et al. Prevalence and predictors of atrial fibrillation in rheumatic valvular heart disease. Am J Cardiol, 1996; 77: 96-98. 
5. Alessandri N, Tufano F, Pertrassi M et al. Atrial fibrillation in pure rheumatic mitral valvular disease is expression of an atrial histological change. Eur Rev Med Pharmacol Sciences, 2009; 13: 431-442.

6. Guilherme L, Fae K, Oshiro SE. Molecular pathogenesis of rheumatic fever and rheumatic heart disease. Expert Rev Mol Med, 2005; 7: 1-15.

7. Deniz A, Yavuz B, Ciftci O et al. Left atrial conduction time detected by tissue Doppler imaging increases in paroxysmal atrial fibrillation patients. Clin Cardiol, 2006; 29 (suppl.): III27.

8. Deniz A, Sahiner L, Aytemir K et al. Tissue Doppler echocardiography can be a useful technique to evaluate atrial conduction time. Cardiol J, 2012; 19: 487-493.

9. Van WagonerDR, Pond AL, Lamorgese M, RossieSS, McCarthy PM, Nerbonne JM. Atrial L-type Ca2+ currents and human atrial fibrillation. Circ Res, 1999; 85: 428-436.

10. Nattel S. Atrial electrophysiological remodeling caused by rapid atrial activation: underlying mechanism and clinical relevance to atrial fibrillation. Cardiovasc Res, 1999; 42: 298-308.

11. Pham TD, Fenoglio JJ Jr. Right atrial ultrastructure in chronic rheumatic heart disease. Int J Cardiol, 1982; 1: 289-304.

12. Perzanowski C, Ho AT, Jacobson AK. Increased P-wave dispersion predicts recurrent atrial fibrillation after cardioversion. J Electrocardiol, 2005; 38: 43-46.

13. Ozer N, Yavuz B, Can I et al. Doppler tissue evaluation of intraatrial and interatrial electromechanical delay and comparison with P-wave dispersion in patients with mitral stenosis. J Am Soc Echocardiogr, 2005; 18: 945-948.
14. Can I, Onat AM, Aytemir K et al. Assessment of atrial conduction in patients with scleroderma by tissue Doppler echocardiography and $\mathrm{P}$ wave dispersion. Cardiology, 2007; 108: 317-321.

15. Tasal A, Guvenc TS, Kul S et al. Atrial conduction abnormalities in patients with psoriasis vulgaris. Kardiol Pol, 2015; 73: 637-643. doi: 10.5603/KP.a2015.0028.

16. Bayés de Luna A, Platonov P, Cosio FG et al. Interatrial blocks. A separate entity from left atrial enlargement: A consensus report. J Electrocardiol, 2012; 45: 445-451

17. Magnani JW, Gorodeski EZ, Johnson VM et al. $P$ wave duration is associated with cardiovascular and all-cause mortality outcomes: the National Health and Nutrition Examination Survey. Heart Rhythm, 2011; 8: 93.

18. Spodick DH, Ariyarajah V. Interatrial block: a prevalent, widely neglected and portentous abnormality. J Electrocardiol, 2008; 41: 61.

19. Dewland TA, Vittinghoff E, Mandyam MC et al. Atrial ectopy as a predictor of incident atrial fibrillation: a cohort study. Ann Intern Med, 2013; 159: 721-728.

20. Chong BH, Pong V, Lam KF et al. Frequent premature atrial complexes predict new occurrence of atrial fibrillation and adverse cardiovascular events. Europace, 2012; 14: 942-947.

21. Sharma S, Lardizabal J, Monterroso M et al. Clinically unrecognized mitral regurgitation is prevalent in lone atrial fibrillation. World J Cardiol, 2012; 26: 183-187.

22. Frustaci A, Chimenti C, Bellocci F et al. Histological substrate of atrial biopsies in patients with lone atrial fibrillation. Circulation, 1997; 96: 1180-1184. 